\title{
EFFECTS OF THE CHEMICAL REACTION AND RADIATION ABSORPTION ON AN UNSTEADY MHD CONVECTIVE HEAT AND MASS TRANSFER FLOW PAST A SEMI-INFINITE VERTICAL MOVING IN A POROUS MEDIUM WITH HEAT SOURCE AND SUCTION
}

\author{
${ }^{1}$ Seethamahalakshmi, ${ }^{2}$ G.V.Ramana Reddy and ${ }^{3}$ B. D. C. N Prasad \\ ${ }^{1,3}$ P.V.P.Siddartha Institute of Technology, Vijayawada, A.P (India)-521007 \\ ${ }^{2}$ Usha Rama College of Engineering and Technology, Telaprolu, A.P (India)-521109
}

\begin{abstract}
Analytical solutions for heat and mass transfer by laminar flow of a Newtonian, viscous, electrically conducting and heat generation/absorbing fluid on a continuously vertical permeable surface in the presence of a radiation, a first-order homogeneous chemical reaction and the mass flux are reported. The plate is assumed to move with a constant velocity in the direction of fluid flow. A uniform magnetic field acts perpendicular to the porous surface, which absorbs the fluid with a suction velocity varying with time. The dimensionless governing Equations for this investigation are solved analytically using two-term harmonic and non-harmonic functions. Graphical results for velocity, temperature and concentration profiles of both phases based on the analytical solutions are presented and discussed.
\end{abstract}

Keywords: MHD, Chemical reaction, Porous medium, vertical plate, Radiation, Skin-friction.

\section{Introduction}

Combined heat and mass transfer problems with chemical reaction are of importance in many processes and have, therefore, received a considerable amount of attention in recent years. In processes such as drying, evaporation at the surface of a water body, energy transfer in a wet cooling tower and the flow in a desert cooler, heat and mass transfer occur simultaneously. Possible applications of this type of flow can be found in many industries. For example, in the power industry, among the methods of generating electric power is one in which electrical energy is extracted directly from a moving conducting fluid. We are particularly interested in cases in which diffusion and chemical reaction occur at roughly the same speed. When diffusion is much faster than chemical reaction, then only chemical factors influence the chemical reaction rate; when diffusion is not much faster than reaction, the diffusion and kinetics interact to produce very different effects. The study of heat generation or absorption effects in moving fluids is important in view of several physical problems, such as fluids undergoing exothermic or endothermic chemical reaction. Due to the fast Growth of electronic technology, effective cooling of electronic equipment has become warranted and cooling of electronic equipment ranges from individual transistors to main frame computers and from energy suppliers to telephone switch boards and thermal diffusion effect has been utilized for isotopes separation in the mixture between gases with very light molecular weight (hydrogen and helium) and medium molecular weight.

Chambre and Young [1] have presented a first order chemical reaction in the neighborhood of a horizontal plate. Dekha et al. [2] investigated the effect of the first order homogeneous chemical reaction on the process of an unsteady flow past a vertical plate with a constant heat and mass transfer. Muthucumaraswamy [3] presented heat and mass transfer effects on a continuously moving isothermal vertical surface with uniform suction by taking into account the homogeneous chemical reaction of first order. Muthucumaraswamy and Meenakshisundaram[4] investigated theoretical study of chemical reaction effects on vertical oscillating plate with variable temperature and mass diffusion.

There has been a renewed interest in studying magnetohydrodynamic (MHD) flow and heat transfer in porous and non-porous media due to the effect of magnetic fields on the boundary layer flow control and on the performance of many systems using electrically conducting fluids. Raptis et al. [5] analyzed hydromagnetic free convection flow through a porous medium between two parallel plates. Gribben [6] presented the boundary layer flow over a semi-infinite plate with an aligned magnetic field in the presence of pressure gradient. $\mathrm{He}$ obtained solutions for large and small magnetic Prandtl number using the method of matched asymptotic expansion. Helmy [7] presented an unsteady two-dimensional laminar free convection flow of an incompressible, electrically conducting (Newtonian or polar) fluid through a porous medium bounded by infinite vertical plane surface of constant temperature. Gregantopoulos et al. [8] studied two-dimensional unsteady free convection and mass transfer flow of an incompressible viscous dissipative and electrically conducting fluid past an infinite vertical porous plate. For some industrial applications such as glass production 
and furnace design, and in space technology applications such as cosmical flight aerodynamics rocket, propulsion systems, plasma physics and spacecraft re-entry aerothermodynamics which operate at higher temperatures, radiation effects can be significant. In view of this, Hossain and Takhar [9] analyzed the effect of radiation on mixed convection along a vertical plate with uniform surface temperature. Kim and Fedorov [10] analyzed transient mixed radiative convective flow of a micropolar fluid past a moving semi-infinite vertical porous plate. Muthuraj and Srinivas [11] studied the fully developed MHD flow of a micropolar and viscous fluid in a vertical porous space using HAM.

The study of heat generation or absorption effects in moving fluids is important in view of several physical problems, such as fluids undergoing exothermic or endothermic chemical reactions. Possible heat generation effects may alter the temperature distribution and consequently, the particle deposition rate in nuclear reactors, electric chips and semiconductor wafers. Seddeek [12] studied the effects of chemical reaction, thermophoresis and variable viscosity on steady hydromagnetic flow with heat and mass transfer over a flat plate in the presence of heat generation/absorption. Patil and Kulkarni [13] studied the effects of chemical reaction on free convective flow of a polar fluid through porous medium in the presence of internal heat generation. Double-Diffusive Convection-Radiation interaction on unsteady MHD flow over a vertical moving porous plate with heat generation and soret effects was studied by Mohamed [14]. Radiation effects on an unsteady MHD convective heat and mass transfer flow past a semi-infinite vertical permeable moving plate embedded in a porous medium was studied by Ramachandraprasad et al. [15]. Satyanarayana et al [16] studied Hall current effect on magneto hydrodynamics free-convection flow past a semi-infinite vertical porous plate with mass transfer. Effects of the chemical reaction and radiation absorption on free convection flow through porous medium with variable suction in the presence of uniform magnetic field were studied by Sudheer Babu and Satyanarayana [17]. Dulal Pal et al [18] studied Perturbation analysis of unsteady magnetohydrodynamic convective heat and mass transfer in a boundary layer slip flow past a vertical permeable plate with thermal radiation and chemical reaction. Recently, Ramana Reddy et al [19] have studied the mass transfer and radiation effects of unsteady MHD free convective fluid flow embedded in porous medium with heat generation/absorption.

In spite of all the previous studies, the unsteady MHD free convection heat and mass transfer for a heat generation/absorption with radiation absorption in the presence of a reacting species over an infinite permeable plate has received little attention. Hence, the main objective of this chapter is to investigate the effects of thermal radiation, chemical reaction, and heat source/sink parameter of an electrically conducting fluid past an infinite vertical porous plate subjected to variable suction. The plate is assumed to be embedded in a uniform porous medium and moves with a constant velocity in the flow direction in the presence of a transverse magnetic field. It is also assumed that temperature over which are superimposed an exponentially varying with time. The Equations of continuity, linear momentum, energy and diffusion, which govern the flow field, are solved by using a regular perturbation method. The behavior of the velocity, temperature, concentration, skinfriction, Nusselt number and Sherwood number has been discussed for variations in the governing parameters.

\section{Mathematical formulation}

We consider unsteady two-dimensional flow of a laminar, viscous, electrically conducting and heatabsorbing fluid past a semi-infinite vertical permeable moving plate embedded in a uniform porous medium and subjected to a uniform transverse magnetic field in the presence of thermal and concentration buoyancy effects. It is assumed that there is no applied voltage which implies the absence of an electrical field. The fluid properties are assumed to be constant except that the influence of density variation with temperature has been considered only in the body-force term. The concentration of diffusing species is very small in comparison to other chemical species, the concentration of species far from the wall, $C_{\infty}$, is infinitesimally small (Dekha [2]) and hence the Soret and Dufour effects are neglected. The chemical reactions are taking place in the flow and all thermophysical properties are assumed to be constant of the linear momentum Equation which is approximated according to the Boussinesq approximation. Due to the semi-infinite plane surface assumption, the flow variables are functions of $y^{*}$ and the time $t^{*}$ only. Under these assumptions, the governing Equations for the problem considered in this chapter are based on the balances of mass, linear momentum, energy and concentration species. These Equations are as given below:

Continuity Equation:

$\frac{\partial v^{\prime}}{\partial y^{\prime}}=0$

Momentum Equation: 
$\frac{\partial u^{\prime}}{\partial t^{\prime}}+v^{\prime} \frac{\partial u^{\prime}}{\partial y^{\prime}}=-\frac{1}{\rho} \frac{\partial p^{\prime}}{\partial x^{\prime}}+g \beta\left(T^{\prime}-T_{\infty}^{\prime}\right)+g \beta^{*}\left(C^{\prime}-C_{\infty}^{\prime}\right)+v \frac{\partial^{2} u^{\prime}}{\partial y^{\prime 2}}-\frac{v}{K^{\prime}} u^{\prime}-\frac{\sigma B_{0}^{2}}{\rho} u^{\prime}$

Energy Equation:

$$
\frac{\partial T^{\prime}}{\partial t^{\prime}}+v^{\prime} \frac{\partial T^{\prime}}{\partial y^{\prime}}=\frac{1}{\rho C_{p}}\left[k \frac{\partial^{2} T^{\prime}}{\partial y^{\prime 2}}-Q_{0}\left(T^{\prime}-T_{\infty}^{\prime}\right)\right]+Q_{l}^{\prime}\left(C^{\prime}-C_{\infty}^{\prime}\right)-\frac{1}{\rho C_{p}} \frac{\partial q_{r}}{\partial y^{\prime}}
$$

Mass diffusion Equation:

$$
\frac{\partial C^{\prime}}{\partial t^{\prime}}+v^{\prime} \frac{\partial C^{\prime}}{\partial y^{\prime}}=D \frac{\partial^{2} C^{\prime}}{\partial y^{\prime 2}}-K_{r}^{\prime}\left(C^{\prime}-C_{\infty}^{\prime}\right)
$$

where $x^{\prime}, y^{\prime}$ are the dimensional distance along and perpendicular to the plate, respectively. $u^{\prime}$ and $v^{\prime}$ are the velocity components in the $x^{\prime}, y^{\prime}$ directions respectively, $\mathrm{g}$ is the gravitational acceleration, $\rho$ is the fluid density, $\beta$ and $\beta^{*}$ are the thermal and concentration expansion coefficients respectively, $K^{\prime}$ is the Darcy permeability, $B_{0}$ is the magnetic induction, $T^{\prime}$ is the thermal temperature inside the thermal boundary layer and $C^{\prime}$ is the corresponding concentration, $\sigma$ is the electric conductivity, $C_{p}$ is the specific heat at constant pressure, $\mathrm{D}$ is the diffusion coefficient, $q_{r}$ is the heat flux, $Q_{0}$ is the dimensional heat absorption coefficient, $Q_{l}^{\prime}$ is the coefficient of proportionality of the radiation and $K_{r}^{\prime}$ is the chemical reaction parameter.

The corresponding boundary conditions for the velocity, temperature and concentration fields are

$$
\begin{aligned}
& u^{\prime}=u_{p}^{\prime}, \quad T^{\prime}=T_{\infty}^{\prime}+\varepsilon\left(T_{w}^{\prime}-T_{\infty}^{\prime}\right) e^{n^{\prime} t^{\prime}}, C^{\prime}=C_{\infty}^{\prime}+\varepsilon\left(C_{w}^{\prime}-C_{\infty}^{\prime}\right) e^{n^{\prime} t^{\prime}} \\
& u^{\prime}=U_{\infty}^{\prime}=U_{0}+\varepsilon\left(1+e^{n^{\prime} t^{\prime}}\right), \quad T^{\prime} \rightarrow T_{\infty}^{\prime}, \quad y^{\prime}=0
\end{aligned}
$$

where $u_{p}^{\prime}$ is the velocity, $T_{w}^{\prime}$ and $C_{w}^{\prime}$ the temperature and concentration of the wall respectively, $U_{\infty}^{\prime}$ - the free stream velocity, and $U_{0}, n^{\prime}$ - the constants. From Equation (1), it is clear that the suction velocity at the plate is either a constant and or a function of time. Hence the suction velocity normal to the plate is assumed in the form

$v^{\prime}=-v_{0}\left(1+\varepsilon A e^{n^{\prime} t^{\prime}}\right)$

where $A$ is a real positive constant, $\varepsilon$ and $\varepsilon A$ is small values less than unity, and $v_{0}$ is scale of suction velocity which is non zero positive constant. The negative sign indicates that the suction is towards the plate. Out side the boundary layer, Equation (2) gives

$$
-\frac{1}{\rho} \frac{\partial p^{\prime}}{\partial x^{\prime}}=\frac{d U_{\infty}^{\prime}}{d t^{\prime}}+\frac{v}{K^{\prime}} U_{\infty}^{\prime}-\frac{\sigma B_{0}^{2}}{\rho} U_{\infty}^{\prime}
$$

By using the Rosseland diffusion approximation and following among other researchers, the radiative heat flux, $q_{r}$ is given by

$$
q_{r}=-\frac{4 \sigma^{*}}{3 K_{s}} \frac{\partial T^{\prime 4}}{\partial y^{\prime}}
$$

Where $\sigma^{*}$ and $K_{s}$ are the Stefan- Boltzmann constant and the Roseland mean absorption coefficient, respectively. We assume that the temperature differences within the flow are sufficiently small such that $T^{4}$ may be expressed as a linear function of temperature.

$T^{4} \approx 4 T_{\infty}^{3} T-3 T_{\infty}^{4}$

Using (8) and (9) in the last term of Equation (3) we obtain 
$\frac{\partial q_{r}}{\partial y^{\prime}}=-\frac{16 \sigma^{*} T_{\infty}^{3}}{3 K_{s}} \frac{\partial^{2} T^{\prime}}{\partial y^{\prime 2}}$

In order to write the governing Equations and the boundary conditions in dimensional following nondimensional Quantities are introduced.

$$
\begin{aligned}
& y=\frac{v_{0} y^{\prime}}{v}, u=\frac{u^{\prime}}{U_{0}}, v=\frac{v^{\prime}}{v_{0}}, t=\frac{t^{\prime} v_{0}^{2}}{v}, U_{\infty}=\frac{U_{\infty}^{\prime}}{U_{0}}, U_{p}=\frac{u_{p}^{\prime}}{U_{0}} \theta=\frac{T^{\prime}-T_{\infty}^{\prime}}{T_{w}^{\prime}-T_{\infty}^{\prime}}, C=\frac{C^{\prime}-C_{\infty}^{\prime}}{C_{w}^{\prime}-C_{\infty}^{\prime}}, \\
& G r=\frac{g \beta v\left(T_{w}^{\prime}-T_{\infty}^{\prime}\right)}{v_{0}^{3}}, G m=\frac{g \beta^{*} v\left(C_{w}^{\prime}-C_{\infty}^{\prime}\right)}{v_{0}^{3}}, S c=\frac{v}{D}, Q=\frac{v Q_{0}}{\rho C_{p} v_{0}^{2}}, Q_{l}=\frac{v Q_{l}^{\prime}\left(C_{w}^{\prime}-C_{\infty}^{\prime}\right)}{\left(T_{w}^{\prime}-T_{\infty}^{\prime}\right) v_{0}^{2}} \\
& n=\frac{v n^{\prime}}{v_{0}^{2}} M=\frac{\sigma B_{0}^{2} v}{\rho v_{0}^{2}} K=\frac{K^{\prime} v_{0}^{2}}{v^{2}}, K_{r}=\frac{K_{r}^{\prime} v}{v_{0}^{2}} R=\frac{4 \sigma^{*} T_{\infty}^{3}}{K_{s}}, \operatorname{Pr}=\frac{v \rho C_{p}}{k}
\end{aligned}
$$

In view of Equations (6) - (11), Equations (2)-(4) reduce to the following dimensional form.

$$
\begin{aligned}
& \frac{\partial u}{\partial t}-\left(1+\varepsilon A e^{n t}\right) \frac{\partial u}{\partial y}=\frac{d U_{\infty}}{d t}+G r \theta+G m C+\frac{\partial^{2} u}{\partial y^{2}}+N\left(U_{\infty}-u\right) \\
& \frac{\partial \theta}{\partial t}-\left(1+\varepsilon A e^{n t}\right) \frac{\partial \theta}{\partial y}=\frac{1}{\operatorname{Pr}}\left(1+\frac{4 R}{3}\right) \frac{\partial^{2} \theta}{\partial y^{2}}-Q \theta+Q_{l} C \\
& \frac{\partial C}{\partial t}-\left(1+\varepsilon A e^{n t}\right) \frac{\partial C}{\partial y}=\frac{1}{S c} \frac{\partial^{2} C}{\partial y^{2}}-K_{r} C
\end{aligned}
$$

where $N=M+\frac{1}{K}, G r, G m, \operatorname{Pr}, K_{r}, S c, Q, Q_{l}$ and $R$ are the magnetic field parameter, permeability parameter, thermal Grashof number, Solutal Grashof number, Prandtl number, Chemical reaction number, Schmidt number, heat absorption parameter, absorption of radiation parameter and thermal radiation parameter.

The corresponding boundary conditions for $t>0$ are transformed to:

$$
\begin{aligned}
& u=U_{p}, \theta=1+\varepsilon e^{n t}, C=1+\varepsilon e^{n t} \quad \text { at } \quad y=0 \\
& u \rightarrow U_{\infty}=1+\varepsilon e^{n t}, \theta \rightarrow 0, C \rightarrow 0 \text { as } \mathrm{y} \rightarrow \infty
\end{aligned}
$$

\section{Solution of the problem}

Equations (12) - (14) are coupled, non - linear partial differential Equations and these cannot be solved in closed form. However, these Equations can be reduced to a set of ordinary differential Equations, which can be solved analytically. This can be done by representing the velocity, temperature and concentration of the fluid in the neighborhood of the fluid in the neighborhood of the plate as

$$
\begin{aligned}
& u(y, t)=u_{0}(y)+\varepsilon e^{n t} u_{1}(y)+o\left(\varepsilon^{2}\right)+\ldots \\
& \theta(y, t)=\theta_{0}(y)+\varepsilon e^{n t} \theta_{1}(y)+o\left(\varepsilon^{2}\right)+. . \\
& C(y, t)=C_{0}(y)+\varepsilon e^{n t} C_{1}(y)+o\left(\varepsilon^{2}\right)+
\end{aligned}
$$

Substituting (16) in Equations (12) - (14) and equating the harmonic and non - harmonic terms, and neglecting the higher order terms of $o\left(\varepsilon^{2}\right)$, we obtain

$$
u_{0}^{\prime \prime}+u_{0}^{\prime}-N u_{0}=-N-G r \theta_{0}-G m C_{0}
$$


Seethamahalakshmi, G.V.Ramana Reddy and B. D. C. N Prasad / IOSR Journal of Engineering (IOSRJEN)

Vol. 1, Issue 1, pp. 028-036

$$
\begin{aligned}
& u_{1}^{\prime \prime}+u_{1}^{\prime}-(N+n) u_{0}=-(N+n)-G r \theta_{1}-G m C_{1}-A u_{0}^{\prime} \\
& \left(1+\frac{4 R}{3}\right) \theta_{0}^{\prime \prime}+\operatorname{Pr} \theta_{0}^{\prime}-\operatorname{Pr} Q \theta_{0}=-\operatorname{Pr} Q_{l} C_{0} \\
& \left(1+\frac{4 R}{3}\right) \theta_{1}^{\prime \prime}+\operatorname{Pr} \theta_{1}^{\prime}-\operatorname{Pr}(Q+n) \theta_{1}=-A \operatorname{Pr} \theta_{0}^{\prime}-\operatorname{Pr} Q_{l} C_{1} \\
& C_{0}^{\prime \prime}+S c C_{0}^{\prime}-S c K_{r} C_{0}=0 \\
& C_{1}^{\prime \prime}+S c C_{1}^{\prime}-S c\left(K_{r}+n\right) C_{1}=-A S c C_{0}^{\prime}
\end{aligned}
$$

where prime denotes ordinary differentiation with respect to $\mathrm{y}$.

The corresponding boundary conditions can be written as

$$
\begin{aligned}
& u_{0}=U_{p}, u_{1}=0, \theta_{0}=1, \theta_{1}=1, C_{0}=1, C_{1}=1 \quad \text { at } y=0 \\
& u_{0} \rightarrow 1, u_{1} \rightarrow 1, \theta_{0} \rightarrow 0, \theta_{1} \rightarrow 0, C_{0} \rightarrow 0, C_{1} \rightarrow 0 \text { as } y \rightarrow \infty
\end{aligned}
$$

Solving Equations (17) - (22) under the boundary condition (23) we obtain the velocity, temperature and concentration distribution in the boundary layer as

$$
\begin{aligned}
& u(y, t)=1+A_{14} \exp \left(-m_{5} y\right)-A_{12} \exp \left(-m_{1} y\right)-A_{13} \exp \left(-m_{3} y\right)+ \\
& \varepsilon \exp (n t)\left[\begin{array}{l}
1+A_{25} \exp \left(-m_{6} y\right)+A_{20} \exp \left(-m_{1} y\right)-A_{21} \exp \left(-m_{2} y\right) \\
-A_{22} \exp \left(-m_{3} y\right)-A_{23} \exp \left(-m_{4} y\right)+A_{24} \exp \left(-m_{5} y\right)
\end{array}\right] \\
& \theta(y, t)=A_{3} \exp \left(-m_{1} y\right)+A_{4} \exp \left(-m_{3} y\right)+\varepsilon \exp (n t)\left[\begin{array}{l}
A_{8} \exp \left(-m_{1} y\right)-A_{9} \exp \left(-m_{2} y\right) \\
+A_{10} \exp \left(-m_{3} y\right)+A_{11} \exp \left(-m_{4} y\right)
\end{array}\right] \\
& C(y, t)=\exp \left(-m_{1} y\right)+\varepsilon \exp (n t)\left[A_{1} \exp \left(-m_{1} y\right)+A_{2} \exp \left(-m_{2} y\right)\right]
\end{aligned}
$$

The skin-friction, Nusselt number and Sherwood number are important physical parameters for this type of boundary layer flow.

\section{Skin friction}

Knowing the velocity field, the skin - friction at the plate can be obtained, which in non -dimensional form is given by

$$
\begin{aligned}
& C_{f}=\frac{\tau_{w}^{\prime}}{\rho U_{0} v_{0}}=\left(\frac{\partial u}{\partial y}\right)_{y=0}=\left(\frac{\partial u_{0}}{\partial y}+\varepsilon e^{n t} \frac{\partial u_{1}}{\partial y}\right)_{y=0} \\
& C_{f}=-\left[-m 5 A_{14}+m_{1} A_{12}+A_{12} m_{3}+\varepsilon e^{n t}\left(\begin{array}{l}
-m_{6} A_{25}-m_{1} A_{20}+A_{21} m_{2} \\
+A_{22} m_{3}+m_{4} A_{23}-A_{24} m_{5}
\end{array}\right)\right]
\end{aligned}
$$

\section{Nusselt number}

Knowing the temperature field, the rate of heat transfer coefficient can be obtained, which in non -dimensional form is given, in terms of the Nusselt number, is given by

$$
\begin{aligned}
& N_{u}=-x \frac{\left(\frac{\partial T^{\prime}}{\partial y^{\prime}}\right)_{y^{\prime}=0}}{\left(T_{w}^{\prime}-T_{\infty}^{\prime}\right)} \Rightarrow N_{u} \operatorname{Re}_{x}^{-1}=\left(\frac{\partial \theta}{\partial y}\right)_{y=0}=\left(\frac{\partial \theta_{0}}{\partial y}+\varepsilon e^{n t} \frac{\partial \theta_{1}}{\partial y}\right)_{y=0} \\
& N_{u} \operatorname{Re}_{x}^{-1}=-m_{1} A_{3}-A_{4} m_{3}+\varepsilon e^{n t}\left[-m_{1} A_{8}+m_{2} A_{9}-m_{3} A_{10}-m_{4} A_{11}\right]
\end{aligned}
$$

where $\operatorname{Re}_{x}=\frac{v_{0} x}{v}$ is the local Reynolds number.

\section{Sherwood number}


Knowing the concentration field, the rate of mass transfer coefficient dimensional form, in terms of the Sherwood number, is given by

$$
\begin{aligned}
& S_{h}=-x \frac{\left(\frac{\partial C^{\prime}}{\partial y^{\prime}}\right)_{y^{\prime}=0}}{\left(C_{w}^{\prime}-C_{\infty}^{\prime}\right)} \Rightarrow S_{h} \operatorname{Re}_{x}^{-1}=\left(\frac{\partial C}{\partial y}\right)_{y=0}=\left(\frac{\partial C_{0}}{\partial y}+\varepsilon e^{n t} \frac{\partial C_{1}}{\partial y}\right)_{y=0} \\
& S_{h} \operatorname{Re}_{x}^{-1}=-\left[-m_{1}+\varepsilon \mathrm{e}^{n t}\left(-m_{1} A_{1}-m_{2} A_{2}\right)\right]
\end{aligned}
$$

can be obtained, which in non -

\section{RESULT AND DISCUSSION}

The effect of magnetic field on velocity profiles in the boundary layer is depicted in Figure (1). From this figure it is seen that the velocity starts from minimum value at the surface and increase till it attains the peak value and then starts decreasing until it reaches to the minimum value at the end of the boundary layer for all the values of magnetic field parameter. It is interesting to note that the effect of magnetic field is to decrease the value of the velocity profiles throughout the boundary layer. The effect of magnetic field is more prominent at the point of peak value i.e. the peak value drastically decreases with increases in the value of magnetic field, because the presence of magnetic field in an electrically conducting fluid introduce a force called the Lorentz force, which acts against the flow if the magnetic field is applied in the normal direction, as in the present problem. This type of resisting force slows down the fluid velocity as shown in this figure. Figure (2) shows the velocity distribution $\boldsymbol{u}$ against $\boldsymbol{y}$ for different values of $\boldsymbol{K} \boldsymbol{r}$. we noticed that the velocity decreases with increases $\boldsymbol{K} \boldsymbol{r}$. The effect of increasing the value of the heat source parameter is to decrease the boundary layer as shown in Figure (3), which is as expected due to the fact that when heat is absorbed the buoyancy force decreases which retards the flow rate and there by giving rise to decrease in the velocity profiles. Figure (4) illustrate the velocity profiles for different values of Prandtl number Pr. The numerical results show that the effect of increasing values of Prandtl number result in decreasing velocity. For the case of different values of thermal Grashof number $\boldsymbol{G r}$, the velocity profiles in the boundary layer are shown in Figure (5). As expected, it is observed that an increase in $\boldsymbol{G r}$ leads to increase in the values of velocity due to enhancement in buoyancy force. Here the positive values of $\boldsymbol{G r}$ correspond to cooling of the surface. Figure (6) presents typical velocity profiles in the boundary layer for various values of the Solutal Grashof number $\mathbf{G m}$, while all other parameters are kept at some fixed values. The velocity distribution attains a distinctive maximum value in the vicinity of the plate surface and then decrease properly to approach the free stream value. As expected, the fluid velocity increases and the peak value more distinctive due to increase in the concentration buoyancy effects represented by $\boldsymbol{G m}$. This is evident in the increase in the value of $\boldsymbol{u}$ as $\boldsymbol{G} \boldsymbol{m}$ increases in Figure (6). Figure (7) display the effects of Schmidt number Sc on velocity profiles. As the Schmidt number increases the velocity filed decreases. This causes the velocity buoyancy effects to decreases yielding a reduction in the fluid velocity. The reductions and the velocity profiles are accompanied by simultaneous reductions in the velocity boundary layers. Figure (8) shows the velocity profiles for different values of the permeability parameter $\boldsymbol{K}$, clearly as $\boldsymbol{K}$ increases the peak values of the velocity tends to increase. Figure (9) shows the velocity profiles for different values of the radiation parameter $\boldsymbol{R}$, clearly as $\boldsymbol{R}$ increases the peak values of the velocity tends to increase. The effect of increasing the value of the absorption parameter $\boldsymbol{Q l}$ on the velocity is shown Figure (10). We observe in this figure that increasing the value of the absorption of the radiation parameter due to increase in the buoyancy force accelerates the flow rate. Figure (11) illustrate the temperature profiles for different values of Prandtl number $\boldsymbol{P r}$. It is observed that the temperature decrease as an increasing the Prandtl number. The reason is that smaller values of $\boldsymbol{P r}$ are equivalent to increase in the thermal conductivity of the fluid and therefore heat is able to diffuse away from the heated surface more rapidly for higher values of $\boldsymbol{P r}$. Hence in the case of smaller Prandtl number the thermal boundary layer is thicker and the rate of heat transfer is reduced. Figure (12) has been plotted to depict the variation of temperature profiles against $\mathbf{y}$ for different values of heat source parameter $\boldsymbol{Q}$ by fixing other physical parameters. From this Graph we observe that temperature decrease with increase in the heat source parameter $\boldsymbol{Q}$ because when heat is absorbed, the buoyancy force decreases the temperature profiles. The effect of absorption of radiation parameter on the temperature profile is shown on Figure (14). It is seen from this figure that the effect of absorption of radiation is to increase temperature in the boundary layer as the radiated heat is absorbed by the fluid which in turn increases the temperature of the fluid very close to the porous boundary layer and its effect diminishes far away from the boundary layer. Figure (15) display the effects of Schmidt number $\boldsymbol{S c}$ on the concentration profiles respectively. As the Schmidt number increases the concentration decreases. Figure 11(b) displays the effects of the chemical reaction $\boldsymbol{K r}$ on concentration profiles. We observe that concentration profiles decreases with increasing $\boldsymbol{K r}$. 
Seethamahalakshmi, G.V.Ramana Reddy and B. D. C. N Prasad / IOSR Journal of Engineering (IOSRJEN)

Vol. 1, Issue 1, pp. 028-036

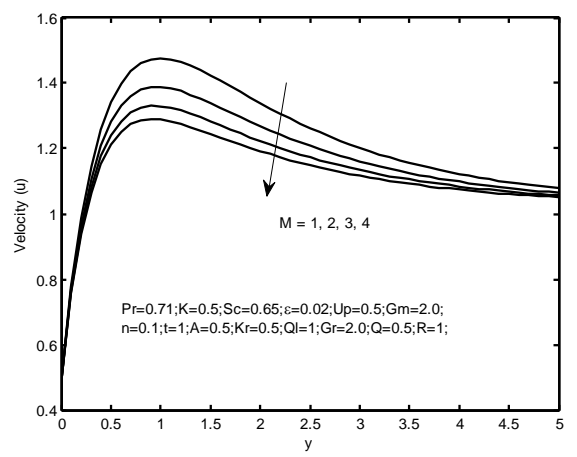

Fig.1.Effects of $\mathbf{M}$ on velocity profiles

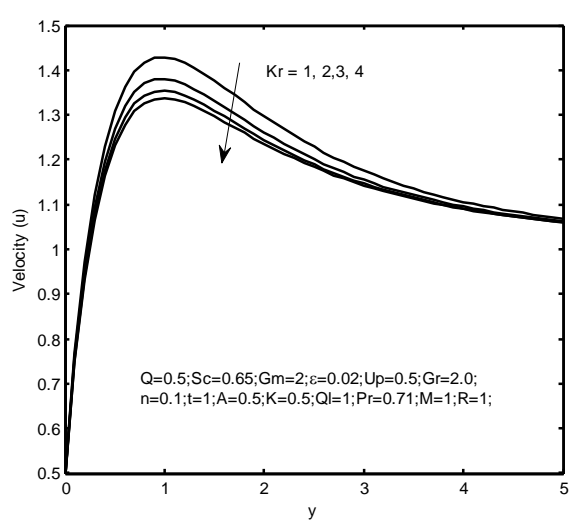

Fig.2.Effects of $\mathbf{K r}$ on velocity profiles.

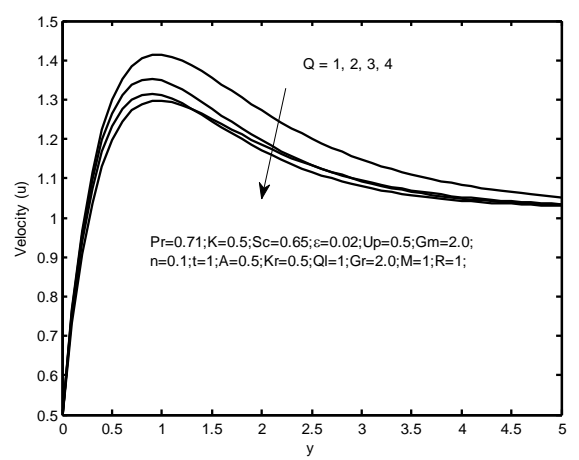

Fig.3.Effects of $\mathbf{Q}$ on velocity profiles.

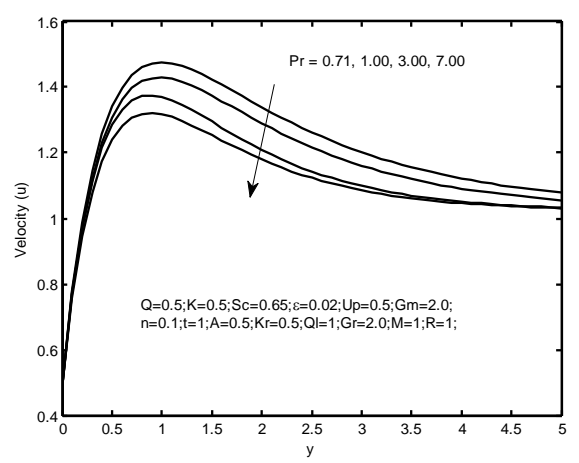

Fig.4.Effects of $\mathbf{P r}$ on velocity profiles.

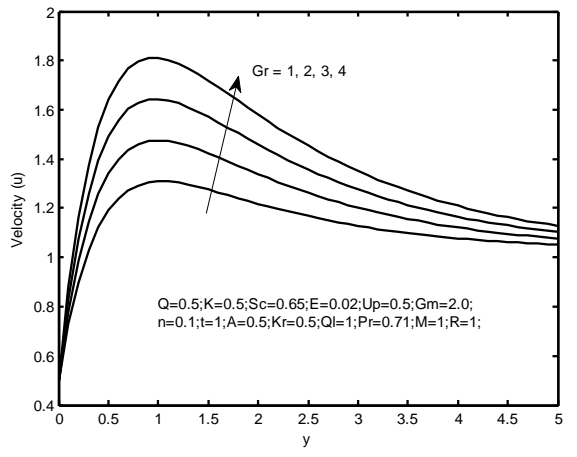

Fig.5.Effects of $\mathbf{G r}$ on velocity profiles.

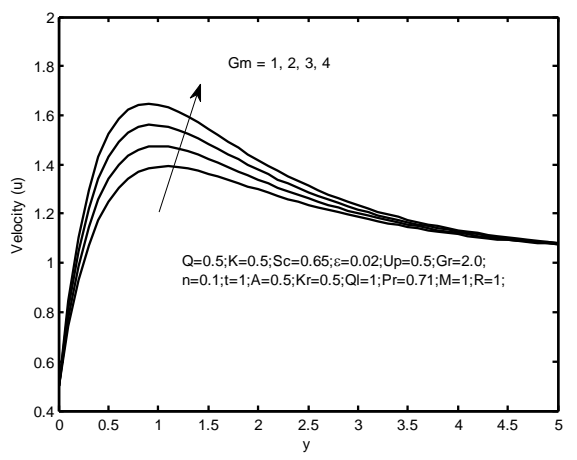

Fig.6.Effects of $\mathrm{Gm}$ on velocity profiles.

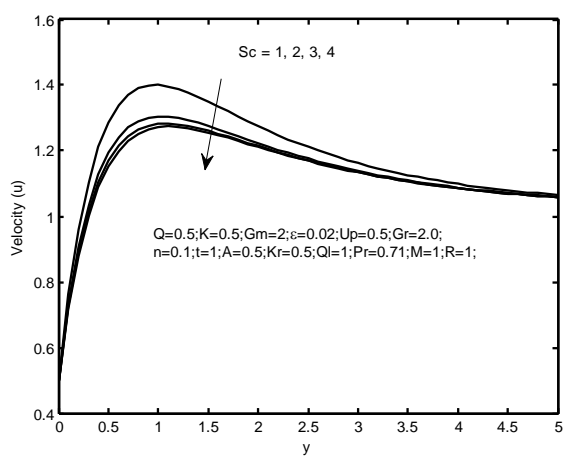

Fig.7.Effects of Sc on velocity profiles.

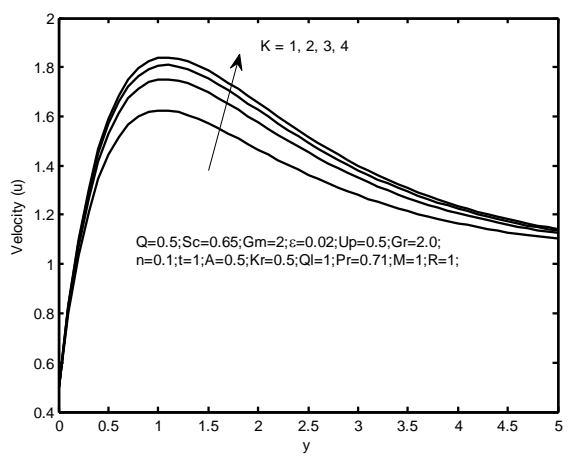

Fig.8.Effects of $\mathbf{K}$ on velocity profiles. 
Seethamahalakshmi, G.V.Ramana Reddy and B. D. C. N Prasad / IOSR Journal of Engineering (IOSRJEN)

Vol. 1, Issue 1, pp. 028-036

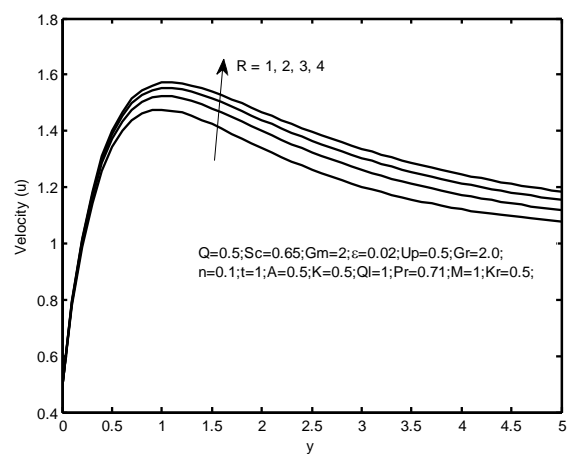

Fig.9. Effects of $\mathbf{R}$ on velocity profiles.

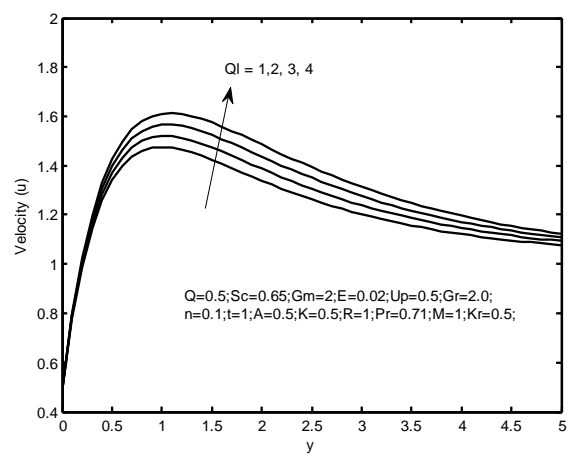

Fig.10. Effects of $\mathbf{Q l}$ on velocity profiles.

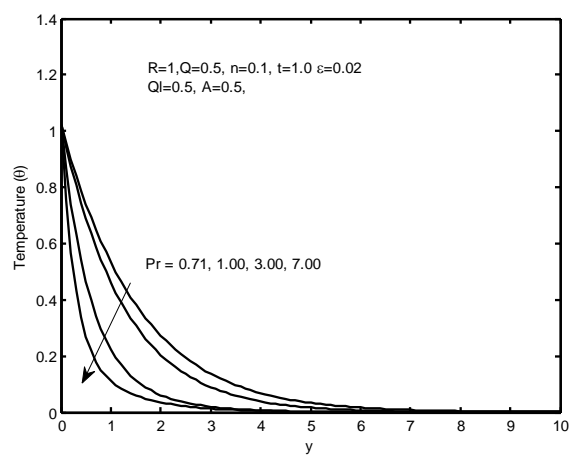

Fig.11. Effects of $\mathbf{P r}$ on temperature profiles.

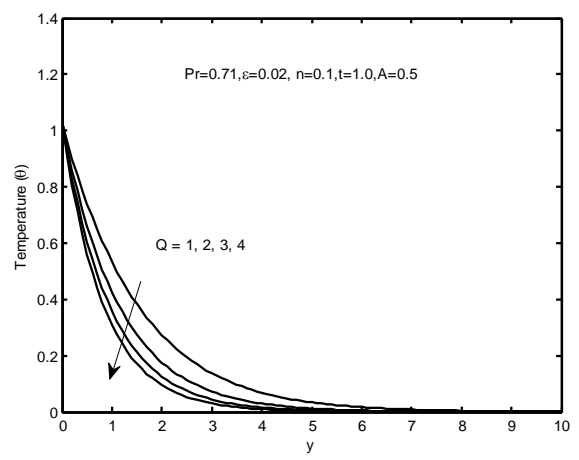

Fig.12. Effects of $\mathbf{Q}$ on temperature profiles.

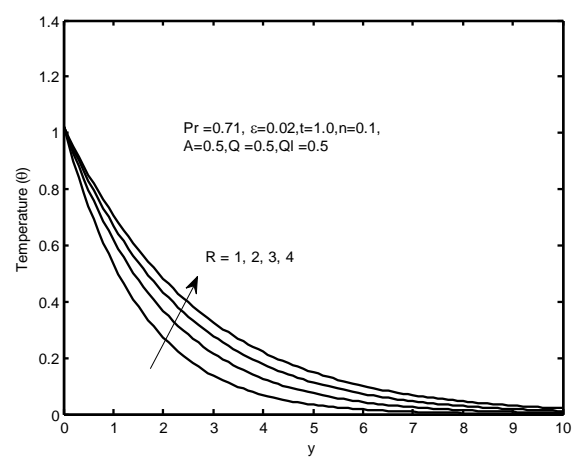

Fig.13. Effects of $\mathbf{R}$ on temperature profiles.

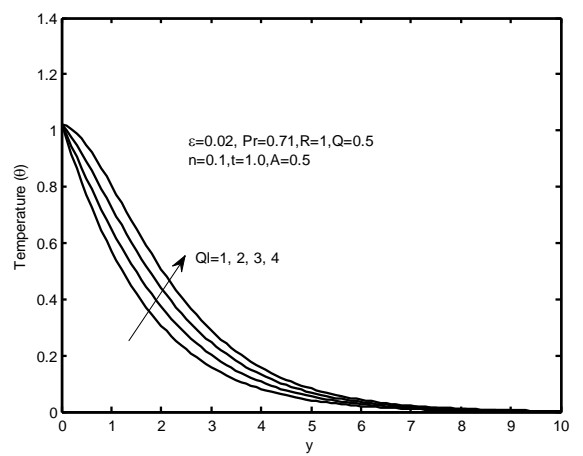

Fig.14. Effects of $\mathbf{Q I}$ on temperature profiles.

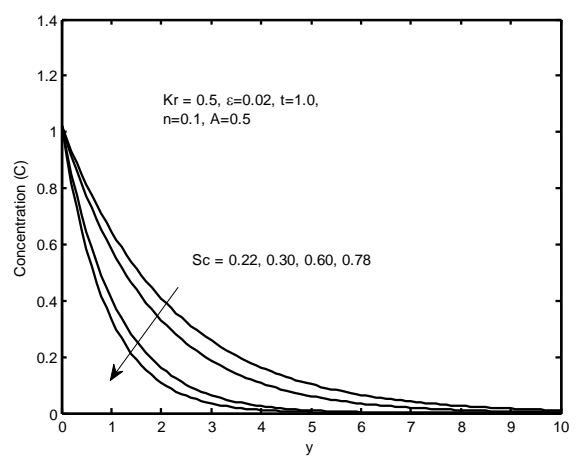

Fig.15. Effects of $\mathbf{S c}$ on concentration profiles.

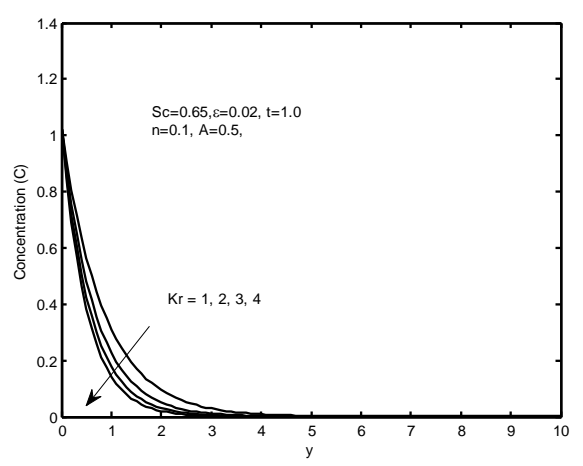

Fig.16. Effects of $\mathbf{K r}$ on concentration profiles.

\section{References}

1. Chambre PL and Young JD (). On the diffusion of a chemically reactive species in a laminar boundary layer flow. Phys. Fluids flow 1, pp. 48-54, 1958. 
2. Dekha R Das UN and Soundalgekar VM (1994). Effects on mass transfer on Flow past an impulsively started infinite vertical plate with constant heat flux and chemical reaction. Forschungim Ingenieurwesen 60, pp. 284-309.

3. Muthecumaraswamy R, (2002). Effects of a chemical reaction on a moving isothermal surface with suction. Acta Mechanica 155, pp.65-72.

4. Muthucumaraswamy R and Meenakshisundaram S (2006). Theoretical study of chemical reaction effects on vertical oscillating plate with variable temperature. Theoret. Appl. Mech 33(3), pp. 245-257.

5. Raptis A, Massalas A and Tzivanidis G (1982). Hydromagnetic free convection flow through a porous medium between two parallel plates, Phys Lett 90A, pp.288-289.

6. Gribben RJ (1965). The magnetohydrodynamic boundary layer in the presence of a pressure Gradient. Proc.Royal.Soc London 287, pp.123-141.

7. Helmy KA (1998). MHD unsteady free convection flow past a vertical porous plate. ZAMM 78, pp.255-270.

8. Gregantopoulos GA, Koullias J, Goudas CL and Courogenis C (1981). Free convection and mass transfer effects on the hydromagnetic oscillatory flow past an infinite vertical porous plate. Astrophysics and space science 74, pp. 357-389.

9. Hossain MA and Takhar HS (1996). Radiation effect on mixed convection along a vertical plate with uniform surface temperature. Heat Mass Transfer 31, pp.243-248.

10. Kim YJ and Fedorov AG (2003). Transient mixed radiative convection flow of a micro polar fluid past a moving semi-infinite vertical porous plate. Int. J.Heat Mass Transfer 46, pp. 1751-1758.

11. Muthuraj R and Srinivas S (2010). Fully developed MHD flow of a micropolar and viscous fluid in a vertical porous space using HAM. Int. J. Appl. Mathematics and Mechanics 6(11), pp. 79-97.

12. Seddek MA (2005). Finite-element Method for the Effects of Chemical Reaction, Variable Viscosity, Thermophoresis and Heat Generation/Absorption on a Boundary-layer Hydro Magnetic Flow with Heat and Mass Transfer Over a Heat Surface. Acta Mech 177, pp. 1-18.

13. Patil, PM and Kulkarni PS (2008). Effects of chemical reaction on free convective flow of a polar fluid through a porous medium in the presence of internal heat generation. Int. J. Therm. Sci 4, pp. 10431054.

14. Mohamad (2009). Double diffusive convection - radiation interaction on unsteady MHD flow over a vertical moving porous plate with heat generation and soret effect was studied. Applied Mathematical Scieces13, pp. 629-651.

15. Ramachandra Prasad V and Bhaskar Reddy N (2008). Radiation effects on an unsteady MHD convective heat and mass transfer flow past a semi-infinite vertical permeable moving plate embedded in a porous medium.Journals of Energy Heat and mass transfer 30, pp.57-68.

16. Satya Narayana PV and Venkataramana S (2007). Hall current effect on magneto hydrodynamics free-convection flow past a semi-infinite vertical porous plate with mass transfer. S.V.University, Ph.D thesis.

17. Sudheer Babu M and Satya Narayana PV (2009). Effects of the chemical reaction and radiation absorption on free convection flow through porous medium with variable suction in the presence of uniform magnetic field, J.P. Journal of Heat and mass transfer 3, pp.219-234.

18. Dulal Pal and Babulal Talukdar (2010). Perturbation analysis of unsteady magneto hydro dynamic convective heat and mass transfer in a boundary layer slip flow past a vertical permeable plate with thermal radiation and chemical reaction. Communications in Nonlinear Science and Numerical Simulation pp.1813-1830.

19. G.V. Ramana Reddy, Ch. V. Ramana Murthy and N. Bhaskar Reddy. Mass transfer and radiation effects of unsteady MHD free convective fluid flow embedded in porous medium with heat generation/absorption, Journal of Applied Mathematics and Fluid Mechanics, vol.2, No.1, pp. 85—98, 2010. 